PROCEEDINGS OF THE

AMERICAN MATHEMATICAL SOCIETY

Volume 136, Number 1, January 2008, Pages 119-126

S 0002-9939(07)09053-3

Article electronically published on September 25, 2007

\title{
COMMON HYPERCYCLIC VECTORS FOR FAMILIES OF OPERATORS
}

\author{
EVA A. GALLARDO-GUTIÉRREZ AND JONATHAN R. PARTINGTON
}

(Communicated by Joseph Ball)

\begin{abstract}
We provide a criterion for the existence of a residual set of common hypercyclic vectors for an uncountable family of hypercyclic operators which is based on a previous one given by Costakis and Sambarino. As an application, we get common hypercyclic vectors for a particular family of hypercyclic scalar multiples of the adjoint of a multiplier in the Hardy space, generalizing recent results by Abakumov and Gordon and also Bayart. The criterion is applied to other specific families of operators.
\end{abstract}

\section{INTRODUCTION}

Let $\mathcal{B}$ be a separable complex Banach space. A bounded linear operator $T$ acting on $\mathcal{B}$ is called cyclic if there is a vector $x \in \mathcal{B}$ such that the linear span generated by its orbit $\left\{x, T x, T^{2} x, \cdots\right\}$ is dense in $\mathcal{B}$. In such a case, the vector $x$ is called cyclic. If the orbit itself is dense, then $T$ is called hypercyclic and $x$ is called a hypercyclic vector for $T$. In what follows, $H C(T)$ will denote the set of hypercyclic vectors for $T$.

Hypercyclic operators and hypercyclic vectors have attracted the attention of many authors in the last decades (see [10] and [11] for a good source of references and results). Let us just mention that the first example of a hypercyclic operator in the Banach space setting was given by Rolewicz [14] in the 1960s. If $B$ denotes the unilateral backward shift $B$ acting on the sequence space $\ell^{p}, 1 \leq p<\infty$, Rolewicz's Theorem states that $\lambda B$ is hypercyclic on $\ell^{p}, 1 \leq p<\infty$, for any complex number $\lambda$ with $|\lambda|>1$.

More recently, questions related to the size of the set of hypercyclic vectors for a family of operators have engaged the attention of many experts in the area. In this sense, it is a well known fact that $H C(T)$ is a $G_{\delta}$ dense set whenever it is nonempty. Hence, as an application of Baire's Theorem, any countable family of hypercyclic operators has a $G_{\delta}$ dense set of common hypercyclic vectors.

Of course, the question of common hypercyclic vectors for an uncountable family of hypercyclic operators is not so straightforward. In this respect, Abakumov and Gordon [1, answering a question posed by Salas [15], have shown the existence of common hypercyclic vectors in $\ell^{2}$ for the family $\{\lambda B:|\lambda|>1\}$.

Received by the editors August 15, 2006.

2000 Mathematics Subject Classification. Primary 47A16; Secondary 47B33, 47B37.

This work was partially supported by Plan Nacional I+D grant no. MTM2006-06431, Gobierno de Aragón research group Análisis Matemático y Aplicaciones, ref. DGA E-64 and a Scheme 4 grant from the London Mathematical Society.

(C)2007 American Mathematical Society 119

Reverts to public domain 28 years from publication 
Bayart [2, Cor. 3], extending Abakumov and Gordon's techniques, has proved that $\{\lambda T:|\lambda|>1\}$ has a common hypercyclic vector, where $T=M_{\phi}^{*}$ is the adjoint of a multiplication operator on the Hardy space $\mathcal{H}^{2}(\mathbb{D})$ by an inner function $\phi$.

In fact, Bayart's result could be seen as a first step towards solving a general question posed by Godefroy and Shapiro [9, Rem. 4.10, Rem. 5.5] in the 1990s, which asks if the scalar multiples of the adjoints of the multipliers which are hypercyclic on a functional Hilbert space have a common hypercyclic vector. We point out that a scalar multiple of the adjoint of a multiplier is hypercyclic if and only if its spectrum meets the unit circle $\mathbb{T}$ (see [9]). Moreover, the family of the adjoints of the multipliers on a functional Hilbert space that are not scalar multiples of the identity has a common cyclic vector (see Bourdon and Shapiro's work [5]).

A preliminary approach to Godefroy and Shapiro's question is the following question raised in the recent paper by Bayart and Grivaux [4, Question 3.3], which still remains open:

Let $\varphi \in \mathcal{H}^{\infty}(\mathbb{D})$ be a multiplier on the Hardy space $\mathcal{H}^{2}(\mathbb{D})$ and define the set $\Lambda$ as $\Lambda=\{\lambda \in \mathbb{C}: \lambda \varphi(\mathbb{D}) \cap \mathbb{T} \neq \emptyset\}$. Does the family $\left\{\lambda M_{\varphi}^{\star}: \lambda \in \Lambda\right\}$ have a common hypercyclic vector?

In this note, we prove a result (Theorem 3.1) which subsumes the Bayart, Abakumov-Gordon and Rolewicz theorems and which in particular yields a positive answer to Bayart and Grivaux's question whenever we suppose that the multiplier $\varphi$ has a nontrivial inner factor and is bounded below on the unit circle $\mathbb{T}$.

To show Theorem 3.1, we prove a criterion for getting common hypercyclic vectors for an uncountable family of hypercyclic operators on a separable Banach space $\mathcal{B}$. Though our criterion is a variant on that one proved by Costakis and Sambarino [7. Theorem 12], we point out that the hypotheses of Costakis and Sambarino's Theorem are not well-adapted to constructing hypercyclic vectors for multiples of an adjoint multiplier. Hence, we first provide in Section 2 an alternative version which is powerful enough for our needs.

In Section 3 we give the application to adjoint multipliers. Another class of operators to which the criterion applies is that of the adjoints of strictly causal multipliers on weighted $\ell^{2}$ spaces. These include certain multipliers on Bergman and Dirichlet spaces. A similar result holds for certain convolution operators on weighted $L^{2}(0, \infty)$ spaces.

We end Section 3 dealing with the class of composition operators. In this sense, Bayart 2] has shown that the family of all invertible composition operators on the Hardy space $\mathcal{H}^{2}(\mathbb{D})$ does not admit a common hypercyclic vector. Here, we will present a family of multiples of adjoints of some composition operators for which it is possible to construct common hypercyclic vectors on the Hardy space.

\section{COMmOn HyPERCYCLIC VECTORS CRITERION}

In [7, Thm. 12], Costakis and Sambarino proved the following result, which they applied to constructing common hypercyclic vectors for certain families of operators.

Theorem (Costakis and Sambarino). Let $\mathcal{B}$ be a separable Banach space and $I$ an open interval of the positive real line. Let $\left\{T_{n, \lambda}: n \in \mathbb{N}, \lambda \in I\right\}$ be a family of operators acting on $\mathcal{B}$ such that for each fixed $n$ the mapping $\lambda \mapsto T_{n, \lambda}$ is continuous. Assume that there is a dense set $\left\{x_{j}: j \geq 0\right\}$ of vectors in $\mathcal{B}$ and a family of operators $\left\{S_{n, \lambda}: n \in \mathbb{N}, \lambda \in I\right\}$ such that $T_{n, \lambda} S_{n, \lambda}=\operatorname{Id}$ and 
(1) given $x_{j}$ and a compact set $K \subset I$, there is a sequence of positive numbers $\left(c_{k}\right)$ such that

(a) $\sum_{k} c_{k}<\infty$,

(b) $\left\|T_{n+k, \lambda} S_{n, \alpha}\left(x_{j}\right)\right\| \leq c_{k}$ for all $n \geq 0, k>0$ and $\lambda, \alpha \in K$ with $\lambda \geq \alpha$,

(c) $\left\|T_{n, \lambda} S_{n+k, \alpha}\left(x_{j}\right)\right\| \leq c_{k}$ for all $n \geq 0, k>0$ and $\lambda, \alpha \in K$ with $\lambda \leq \alpha$,

(2) given $\epsilon>0, x_{j}$ and a compact set $K \subset I$, there exists $0<C(\epsilon)<1$ such that for $\lambda, \alpha \in K$ the following holds: if $1 \geq \lambda / \alpha>C(\epsilon)^{1 / n}$, then $\left\|T_{n, \lambda} S_{n, \alpha}\left(x_{j}\right)-x_{j}\right\|<$ $\epsilon$.

Then there is a residual set $G$ of vectors of $\mathcal{B}$ such that for every $\lambda \in I$ and $x \in G$ the set $\left\{T_{n, \lambda}(x): n \geq 0\right\}$ is dense in $\mathcal{B}$.

Assumption 1(b) is stated in [7] as holding for all $\lambda$ and $\alpha$ in $K$; this is not necessary, as the proof uses only the case $\lambda \geq \alpha$. In [4], Bayart and Grivaux quoted the result with this weaker assumption.

We shall often be interested in the case $T_{n, \lambda}=(\lambda T)^{n}$, where $T$ is a fixed operator; in that case $G$ consists of vectors that are hypercyclic for each $\lambda T$. By [7, Theorem 16], it is sufficient to consider real positive $\lambda$ as: if $x$ is hypercyclic for $\lambda T$, then it is hypercyclic for $\lambda e^{2 \pi i t} T$ for any $t \in[0,1]$.

As mentioned in the introduction, the hypotheses of Costakis and Sambarino's Theorem are not well-adapted to constructing hypercyclic vectors for multiples of an adjoint multiplier, and our first step is to provide the following alternative version of the theorem

Theorem 2.1. The conclusions of Costakis and Sambarino's Theorem remain true if we replace hypothesis $(1)(b)$ by

(1)( $\left.b^{\prime}\right)$ there is an integer $k_{j}$ such that, whenever $k \geq k_{j}$, one has $T_{k, \lambda}\left(x_{j}\right)=0$ and also, for all $n \geq 0$ and $\lambda, \alpha \in K$ with $\lambda \geq \alpha$, one has $T_{n+k, \lambda} S_{n, \alpha}\left(x_{j}\right)=0$.

Note that conditions $(1)(b)$ and $(1)\left(b^{\prime}\right)$ are in general inequivalent, but that $(1)\left(b^{\prime}\right)$ is often satisfied in applications to specific operators and that $(1)(b)$ is almost never satisfied if we have $T_{n, \lambda}=(\lambda T)^{n}$.

Proof. The proof is a modification of the proof of Theorem 12 of [7], and we highlight the changes needed when we assume $(1)\left(b^{\prime}\right)$ rather than $(1)(b)$. With $K=\left[\lambda_{1}, \lambda_{2}\right] \subset I$ we define

$$
E_{K}(s, j, m)=\left\{x \in \mathcal{B}: \forall \lambda \in K \exists n=n(\lambda) \leq m \text { such that }\left\|T_{n, \lambda}(x)-x_{j}\right\|<1 / s\right\} .
$$

As in [7] the sets $E_{K}(s, j, m)$ are open and, therefore, it is only necessary to show that $\bigcup_{m} E_{K}(s, j, m)$ is dense.

Let $w=x_{p}$ for some $p \in \mathbb{N}$ and $\delta>0$. Then $T_{n, \lambda} w=0$ for $n \geq k=k_{p}$ and for every $\lambda \in K$. Define

$$
y=w+S_{k, \alpha_{0}}\left(x_{j}\right)+S_{2 k, \alpha_{1}}\left(x_{j}\right)+\ldots+S_{(l+1) k, \alpha_{l}}\left(x_{j}\right),
$$

where $\lambda_{1}=\alpha_{0}<\alpha_{1}<\ldots<\alpha_{l}=\lambda_{2}$ is a partition of $\left[\lambda_{1}, \lambda_{2}\right]$. 
Now, $\|y-w\|<c_{k}+c_{2 k}+\ldots<\delta$, for $k$ sufficiently large, and for $\lambda$ with $\alpha_{i-1}<\lambda \leq \alpha_{i}$, we have

$$
\begin{aligned}
T_{(i+1) k, \lambda}(y)-x_{j} & =a+b+c+d, \quad \text { where } \\
a & =T_{(i+1) k, \lambda}(w), \\
b & =T_{(i+1) k, \lambda} S_{k, \alpha_{0}}\left(x_{j}\right)+\ldots+T_{(i+1) k, \lambda} S_{i k, \alpha_{i-1}}\left(x_{j}\right), \\
c & =T_{(i+1) k, \lambda} S_{(i+1) k, \alpha_{i}}\left(x_{j}\right)-x_{j}, \quad \text { and } \\
d & =T_{(i+1) k, \lambda} S_{(i+2) k, \alpha_{i+1}}\left(x_{j}\right)+\ldots+T_{(i+1) k, \lambda} S_{(l+1) k, \alpha_{l}}\left(x_{j}\right) .
\end{aligned}
$$

By choosing $k$ sufficiently large, we have that $a=b=0$, by (1)( $\left.b^{\prime}\right)$, and $\|d\|<$ $c_{k}+c_{2 k}+\ldots<\frac{1}{2 s}$ by $(1)(c)$. We also have $\|c\|<\frac{1}{2 s}$ using (2), provided that the partition is sufficiently fine.

We conclude that for these choices we have $\|y-w\|<\delta$ and

$$
\left\|T_{(i+1) k, \lambda}(y)-x_{j}\right\|<1 / s,
$$

as required. Hence, the statement of Theorem 2.1 follows.

Remark 2.1. Note that, as in the Hypercyclicity Criterion (see [9, Corollary 1.5], for instance) the proof of Costakis and Sambarino's Theorem still works if it is not assumed that the maps $S_{n, \lambda}$, with $n \in \mathbb{N}$ and $\lambda \in I$, are linear or continuous (or even defined everywhere).

\section{Applications to SPECific OPERATORS}

3.1. Adjoint multipliers on Hardy spaces. We apply Theorem 2.1 to prove the following result on common hypercyclic vectors for the hypercyclic scalar multiples of the adjoint of a multiplier in the Hardy space $\mathcal{H}^{2}(\mathbb{D})$

Theorem 3.1. Suppose that $\phi \in \mathcal{H}^{\infty}(\mathbb{D})$ is bounded below on $\mathbb{T}$ but is not an outer function. Then the set $\left\{\lambda M_{\phi}^{\star}:|\lambda|>\|1 / \phi\|_{L^{\infty}(\mathbb{T})}\right\}$ has a residual set of common hypercyclic vectors in $\mathcal{H}^{2}(\mathbb{D})$.

Proof. In the notation of Theorem 2.1, we write $T=M_{\phi}^{\star}$ and $T_{n, \lambda}=(\lambda T)^{n}$. Let the inner-outer factorization of $\phi$ be $\phi=u \theta$, where $u$ is outer and invertible in $\mathcal{H}^{\infty}(\mathbb{D})$ and $\theta$ is inner and nonconstant.

Write $K_{n}=\mathcal{H}^{2}(\mathbb{D}) \ominus \theta^{n} \mathcal{H}^{2}(\mathbb{D})$. Clearly $\bigcup_{n=1}^{\infty} K_{n}$ is a dense subspace of $\mathcal{H}^{2}(\mathbb{D})$, as otherwise there is a nonzero vector $v$ orthogonal to each $K_{n}$. That is, $v \in$ $\theta^{n} \mathcal{H}^{2}(\mathbb{D})$ for each $n$, which is impossible. We may therefore take a countable dense set $\left\{x_{j}: j \geq 0\right\}$ with each $x_{j}$ lying in some $K_{N(j)}$. Then

$$
\left\langle T^{N_{j}} x_{j}, y\right\rangle=\left\langle x_{j}, u^{N_{j}} \theta^{N_{j}} y\right\rangle=0,
$$

for each $y \in \mathcal{H}^{2}(\mathbb{D})$, so that $T^{N_{j}} x_{j}=0$.

Define $S=M_{1 / u}^{*} M_{\theta}$. We deduce that

$$
\langle T S x, y\rangle=\langle S x, \phi y\rangle=\left\langle\theta x, u^{-1} u \theta y\right\rangle=\langle x, y\rangle,
$$

for all $x, y \in H^{2}$ since $\theta$ is inner. Write $S_{n, \lambda}=\left(\lambda^{-1} S\right)^{n}$, so that $T_{n, \lambda} S_{n, \lambda}=\mathrm{Id}$.

Now given $x_{j} \in K_{N(j)}$ and a compact interval $K=[a, b]$ with $a>\|1 / \phi\|_{\infty} \geq$ $\|S\|$, we clearly have condition $(1)\left(b^{\prime}\right)$, and also

$$
T_{n, \lambda} S_{n+k, \alpha}\left(x_{j}\right)=\frac{\lambda^{n}}{\alpha^{n+k}} S^{k} x_{j},
$$


so that

$$
\left\|T_{n, \lambda} S_{n+k, \alpha}\left(x_{j}\right)\right\| \leq \frac{\left\|S^{k} x_{j}\right\|}{|\alpha|^{k}} \quad(\lambda, \alpha \in K, \quad \lambda \leq \alpha)
$$

which gives condition (1)(c) with $c_{k}=\left\|S^{k}\right\|\left\|x_{j}\right\| / a^{k}$, which satisfies (1)(a).

Finally, condition (2) holds since $T_{n, \lambda} S_{n, \alpha}=(\lambda / \alpha)^{n} \mathrm{Id}$. Hence, by Theorem 2.1, the set $\left\{\lambda T:|\lambda|>\|1 / \phi\|_{L^{\infty}(\mathbb{T})}\right\}$ has a common hypercyclic vector.

Surprisingly, it is still unknown whether there is a common hypercyclic vector for the set $\left\{\lambda M_{\phi}^{*}:|\lambda|>1 / 2\right\}$, when $\phi$ is the outer function $z \mapsto z+1$. Note that the operator $M_{\phi}^{*}$ is simply $I+B$, where $B$ is the backward shift.

3.2. Adjoint convolution operators. Let $w=\left(w_{n}\right)_{n=0}^{\infty}$ be a strictly positive weight sequence and let $\ell^{2}(w)$ denote the Hilbert space of complex sequences $a=$ $\left(a_{n}\right)_{n=0}^{\infty}$ such that

$$
\left\|\left(a_{n}\right)\right\|_{w}^{2}:=\sum_{n=0}^{\infty}\left|a_{n}\right|^{2} w_{n}<\infty .
$$

We suppose as usual that $\sup _{n} w_{n+1} / w_{n}<\infty$, so that the right shift is a bounded operator on $\ell^{2}(w)$. In many cases it is convenient to identify the sequence $\left(a_{n}\right)$ with the power series $\sum_{n=0}^{\infty} a_{n} z^{n}$, in which case the shift corresponds to multiplication by the independent variable $z$. Two cases of particular interest are the sequences with $a_{n}=1 /(n+1)$ and $a_{n}=\max (1, n)$, which correspond to the well-known Bergman and Dirichlet spaces, respectively (see, for example, 17]). A multiplier $T$ on the space $\ell^{2}(w)$ is a bounded operator that commutes with the right shift, and it is easily seen to be given by a convolution:

$$
(T a)_{n}=\sum_{k=0}^{n} c_{k} a_{n-k},
$$

for some sequence $\left(c_{k}\right)_{k=0}^{\infty}$. Borrowing the language of linear systems, the convolution operator $T$ is said to be strictly causal if $c_{0}=0$, so that $(T a)_{n}$ depends only on $(T a)_{k}$ for $0 \leq k<n$.

Theorem 3.2. Let $T$ be a strictly causal convolution operator on $\ell^{2}(w)$, such that for some $\beta>0$ one has $\|T x\| \geq \beta\|x\|$, for all $x \in \ell^{2}(w)$. Then the set $\left\{\lambda T^{*}:|\lambda|>\right.$ $\left.\beta^{-1}\right\}$ has a residual set of common hypercyclic vectors.

Proof. We write $T_{n, \lambda}=\left(\lambda T^{*}\right)^{n}$. Since $T$ is bounded below, it has a left inverse $S$ (i.e., $S T$ is the identity) such that $\|S\| \leq \beta^{-1}$; we define $S_{n, \lambda}=\left(\lambda^{-1} S^{*}\right)^{n}$ and note that for $n, k \geq 0$ and $\alpha, \lambda>0$ we have

$$
T_{n+k} S_{n, \alpha}=\lambda^{n+k} \alpha^{-n}\left(T^{*}\right)^{k} \quad \text { and } \quad T_{n, \lambda} S_{n+k, \alpha}=\lambda^{n} \alpha^{-n-k}\left(S^{*}\right)^{k} .
$$

We now take a countable dense set of vectors $\left(x_{j}\right)$, each of which has finite support; thus, since $T$ is strictly causal, for each such $j$ there is an index $N(j)$ such that $\left\langle x_{j}, T^{N(j)} y\right\rangle=0$ for all $y$, and hence $\left(T^{*}\right)^{N(j)} x_{j}=0$. As in the proof of Theorem 3.1] we see easily that Conditions (1) (a)-( $\left.\mathrm{b}^{\prime}\right)-(\mathrm{c})$ and (2) of Section 2 are satisfied, and the result now follows from Theorem 2.1 . 
A similar result holds in the weighted Lebesgue space $L^{2}((0, \infty) ; w)$, where now $w$ is a positive measurable locally bounded function, and the functions in the space satisfy

$$
\|f\|_{w}^{2}=\int_{0}^{\infty}|f(t)|^{2} w(t) d t<\infty
$$

with, as usual, functions identified if they are equal almost everywhere. A strictly causal convolution operator now has the form

$$
(T f)(t)=\int_{0}^{t} f(t-\tau) d \mu(\tau)
$$

for a distribution $\mu$ supported on $[\sigma, \infty)$ for some $\sigma>0$. (For example, a right shift corresponds to a Dirac distribution.) In many cases this corresponds to a multiplication operator on a space of Laplace transforms: for instance, in the unweighted case, the space of transforms is a Hardy space on the right half-plane (see, for example, [13, Sec. 1.2]).

Theorem 3.3. Let $T$ be a strictly causal convolution operator on $L^{2}((0, \infty) ; w)$, such that for some $\beta>0$ one has $\|T x\| \geq \beta\|x\|$, for all $x \in L^{2}((0, \infty) ; w)$. Then the set $\left\{\lambda T^{*}:|\lambda|>\beta^{-1}\right\}$ has a residual set of common hypercyclic vectors.

Proof. The proof is similar to that of Theorem 3.2, where now the dense set $\left(x_{j}\right)$ is taken to consist of functions of compact support.

Remark 3.1. Note that the weight $w$ in both results Theorem 3.2 and Theorem 3.3 plays a role in ensuring that $T$ is bounded as well as bounded below.

3.3. Composition operators. Recall that if $\phi$ is an analytic function on the unit disc $\mathbb{D}$ with $\phi(\mathbb{D}) \subset \mathbb{D}$, then the equation

$$
C_{\phi} f=f \circ \phi
$$

defines a composition operator $C_{\phi}$ on the space of all holomorphic functions on the unit disc $\mathcal{H}(\mathbb{D})$. Moreover, Littlewood's Subordination Principle [12] states that $C_{\phi}$ acts boundedly on the Hardy space $\mathcal{H}^{2}(\mathbb{D})$. For more details on the subject, we refer the reader to 8,16 .

As mentioned in the introduction, Bayart 2 has shown that the family of all invertible composition operators on the Hardy space $\mathcal{H}^{2}(\mathbb{D})$ does not admit a common hypercyclic vector. Nevertheless, in 4 ] it is shown that the family of composition operators induced by disc automorphisms having +1 as attractive fixed point has a residual set of common hypercyclic vectors in $\mathcal{H}^{2}(\mathbb{D})$. Here, we present a family of multiples of adjoints of some composition operators for which it is possible to construct common hypercyclic vectors on $\mathcal{H}^{2}(\mathbb{D})$. In what follows, we denote by $\mathcal{H}_{0}^{2}(\mathbb{D})$ the reducing subspace $\mathcal{H}_{0}^{2}(\mathbb{D})=\left\{f \in \mathcal{H}^{2}(\mathbb{D}): f(0)=0\right\}$.

Proposition 3.1. Let $\phi$ be an inner function such that $\phi(0)=0$ and $\phi$ is not the identity function. Then the family $\left\{\lambda C_{\phi}^{*}:|\lambda|>1\right\}$ acting on $\mathcal{H}_{0}^{2}(\mathbb{D})$ has a residual set of common hypercyclic vectors.

Proof. The key point of the proof relies on the fact that $C_{\phi}$ is a unilateral shift of multiplicity $\operatorname{dim}\left(\mathcal{H}_{0}^{2}(\mathbb{D}) \ominus C_{\phi} \mathcal{H}_{0}^{2}(\mathbb{D})\right.$ ) (see, for example, [6]). Hence, upon applying Theorem 2.1. it is possible to construct a common hypercyclic vector for all $\lambda C_{\phi}^{*}$ with $|\lambda|>1$. 
Remark 3.2. We point out that Bayart's result [2, Cor. 3] on $\lambda M_{\phi}^{*}$, where $\phi$ is inner, is a particular case of Proposition 3.1, since these operators $M_{\phi}$ are also unitarily equivalent to shifts.

To end the section, we note more generally that if $w=\left(w_{n}\right)_{n=0}^{\infty}$ is a strictly positive weight sequence as in Section 3.2, satisfying also $\eta_{N}:=\inf _{n} w_{N+n} / w_{n}>0$ for every $N \geq 1$, and if $\mathcal{H}^{2}(w)$ denotes the functional version of $\ell^{2}(w)$, that is, the Hilbert space of analytic functions $f=\sum_{n=0}^{\infty} a_{n} z^{n}$, defined on an appropriate disc $R \mathbb{D}$ with $0<R \leq 1$, such that

$$
\|f\|_{w}^{2}:=\sum_{n=0}^{\infty}\left|a_{n}\right|^{2} w_{n}<\infty
$$

then the composition operator $C_{\phi}$ induced by $\phi(z)=z^{N}$, with $N \geq 1$, is bounded below in $\mathcal{H}^{2}(w)$, namely we always have $\left\|C_{\phi} f\right\|_{w} \geq \eta_{N}^{1 / 2}\|f\|_{w}$. Therefore, its adjoint has a bounded right inverse and as an application of Theorem 2.1 we may deduce the following:

Proposition 3.2. Let $\phi(z)=z^{N}$ with $N \geq 2$. Then the family of operators $\left\{\lambda C_{\phi}^{*}:|\lambda|>\eta_{N}^{-1 / 2}\right\}$ acting on $\mathcal{H}_{0}^{2}(w)=\left\{f \in \mathcal{H}^{2}(w): f(0)=0\right\}$ has a residual set of common hypercyclic vectors.

\section{ACKNOWLEDGEMENTS}

Part of this work was done while the first author was visiting the University of Leeds. She is grateful to the School of Mathematics at the University of Leeds for its hospitality. The authors also wish to thank the London Mathematical Society for financial support and the referee for helpful comments.

\section{REFERENCES}

[1] E. Abakumov and J. Gordon, Common hypercyclic vectors for multiples of backward shift, J. Funct. Anal., 200, (2003), no. 2, 494-504. MR1979020 (2004g:47012)

[2] F. Bayart, Common hypercyclic vectors for composition operators, J. Operator Theory, 52, (2004), no. 2, 353-370. MR2119275 (2006a:47014)

[3] F. Bayart and S. Grivaux, Hypercyclicité: le rôle du spectre ponctuel unimodulaire C. R. Math. Acad. Sci. Paris, 338, (2004), no. 9, 703-708. MR2065378(2005c:47009)

[4] F. Bayart and S. Grivaux, Hypercyclicity and unimodular point spectrum, J. Funct. Anal., 226, (2005), no. 2, 281-300. MR.2159459(2006i:47014)

[5] P. S. Bourdon and J. H. Shapiro, Spectral synthesis and common cyclic vectors, Michigan Math. J., 37, (1990), no. 1, 71-90. MR.1042515 (91m:47039)

[6] I. Chalendar and J. R. Partington, On the structure of invariant subspaces for isometric composition operators on $H^{2}(\mathbb{D})$ and $H^{2}\left(\mathbb{C}_{+}\right)$, Arch. Math. (Basel), 81, (2003), no. 2, 193207. MR2009562 (2004g:47030)

[7] G. Costakis and M. Sambarino, Genericity of wild holomorphic functions and common hypercyclic vectors, Adv. Math., 182, (2004), no. 2, 278-306. MR.2032030 (2004k:47009)

[8] C. C. Cowen and B. D. MacCluer, Composition Operators on Spaces of Analytic Functions, CRC Press, 1995. MR1397026 (97i:47056)

[9] G. Godefroy and J. H. Shapiro, Operators with dense, invariant, cyclic vector manifolds, J. Funct. Anal., 98, (1991), no. 2, 229-269. MR1111569 (92d:47029)

[10] K. G. Grosse-Erdmann, Universal families and hypercyclic operators, Bull. Amer. Math. Soc (NS), 36, (1999), 345-381. MR:1685272 (2000c:47001)

[11] K. G. Grosse-Erdmann, Recent developments in hypercyclicity, RACSAM Rev. R. Acad. Cienc. Exactas Fís. Nat. Ser. A Mat., 97, (2003), no. 2, 273-286. MR2068180(2005c:47010)

[12] J. E. Littlewood, On inequalities in the theory of functions, Proc. London Math. Soc., 23, (1925) 481-519. 
[13] J.R. Partington, Linear operators and linear systems, London Mathematical Society Student Texts, 60. Cambridge University Press, Cambridge, 2004. MR2158502 (2006d:93001)

[14] S. Rolewicz, On orbits of elements, Studia Math., 32, (1969), 17-22. MR0241956 (39:3292)

[15] H. Salas, Supercyclicity and weighted shifts, Studia Math., 135, (1999), no. 1, 55-74. MR1686371 (2000b:47020)

[16] J. H. Shapiro, Composition Operators and Classical Function Theory, Springer-Verlag, Berlin, 1993. MR.1237406 (94k:47049)

[17] K.H. Zhu, Operator theory in function spaces, Marcel Dekker, Inc., New York, 1990. MR:1074007 (92c:47031)

Departamento de Matemáticas, Universidad de Zaragoza e iUma, Plaza San FranCisco S/n, 50009 Zaragoza, Spain

E-mail address: eva@unizar.es

School of Mathematics, University of Leeds, Leeds LS2 9JT, United Kingdom

E-mail address: J.R.Partington@leeds.ac.uk 